\title{
Exosome: An Emerging Participant in the Development of Liver
}

\section{Disease}

\author{
Pengyi Guo, ${ }^{1}$ Haitao Yu, ${ }^{1}$ Yi Wang, ${ }^{2}$ Xiaozai Xie, ${ }^{1}$ and Gang Chen ${ }^{1,}$ \\ ${ }^{1}$ Department of Hepatobiliary Surgery, the First Affiliated Hospital, Wenzhou Medical University, Wenzhou, China \\ ${ }^{2}$ Department of Preventive Medicine, Wenzhou Medical University, Wenzhou, China \\ "Corresponding author: Gang Chen, Department of Hepatobiliary Surgery, The First Affiliated Hospital, Wenzhou Medical University, Wenzhou, China. Tel: +86-057755979555, \\ Fax: +86-057755579453, E-mail: chen.gang@wmu.edu.cn \\ Received 2016 November 27; Accepted 2017 July 04.
}

\begin{abstract}
Context: An exosome is a type of extracellular vesicle with a diameter of $30-100 \mathrm{~nm}$ and a density of $1.13-1.19 \mathrm{~g} / \mathrm{m}$. Exosomes exist in almost all body fluids and can be secreted and absorbed by most cells. They deliver information and molecules that participate in intracellular communication, thus contributing to the progression of various diseases, such as liver diseases.

Evidence Acquisition: In this study we collected and summarized the most important and new data available on the role of exosome in liver diseases by the PubMed search. The study data were collected through searching the related keywords then classified and summarized.

Results: In this review, we summarize the research findings regarding the roles of exosomes in liver physiology and pathology, mainly focusing on liver diseases such as viral hepatitis, liver cancer (mostly hepatocellular carcinoma [HCC]), and liver injury caused by other pathogenic factors (alcohol, drugs, hepatotoxins, high-fat diets, parasites, etc.).

Conclusions: These studies revealed the involvement of exosomes in various aspects of liver physiology and pathology and in the progress of liver diseases. More importantly, it offers a promising new direction for disease diagnosis and treatment.
\end{abstract}

Keywords: Exosomes, Liver Diseases, Hepatocellular Carcinoma, Biomarkers

\section{Context}

Exosomes can act as natural shuttles to deliver bioactive molecules that alter the behavior of the recipient cells, offering a new means of cell-cell communication in physiological and pathological processes in tissues or organs. Thus, exosomes and their contents may be used as novel potential biological markers for disease diagnosis and prognosis, as a vehicle for drug intervention and RNA interference therapy, or as a potential target for clinical treatment. For instance, exosomes play a functional role in myocardial remodeling (1), Alzheimer disease (AD) (2), breast cancer $(3,4)$, and other cancers (5-7).

\subsection{Exosomes}

Exosomes are extracellular vesicles of the endocytic origin with a lipid-bilayer membrane (8). Johnstone et al. discovered the small vesicles of endosomal origin that are released during reticulocyte differentiation and named “exosomes" (9). At present, exosomes are defined according to certain characteristic criteria, including a size between 30 and $100 \mathrm{~nm}(10,11)$, a density of $1.13-1.19 \mathrm{~g} / \mathrm{mL}$ $(6,12)$, specific morphological features (i.e., a cup- or dishshaped morphology when observed with transmission electron microscopy (6), or a round shape when observed with cryo-electron microscopy $(8,11)$, and having general characteristic protein components (CD63 and TGS101).

Exosomes carry a variety of contents, including various proteins, DNA, mRNA, microRNA (miRNA), long noncoding RNA (IncRNA), and even viruses. According to the ExoCarta database (http://www.exocarta.org/), the presence of 41,860 proteins, more than 7540 RNA molecules and 1116 lipid molecules has been confirmed in exosomes in more than 286 studies (13).

In the human body, exosomes have been isolated from various bodily fluids. They can be secreted and ingested by a wide variety of cells $(8,14)$. After the exosomes arrive at their destination, the information is transmitted from the exosomes to the recipient cells in 1 of 3 ways: 1 ) through a ligand-receptor interaction on the cell membrane surface $(15) ; 2)$ through the fusion of the exosome membrane with the recipient cell membrane (16); and 3) through an internalization of exosomes via cellular endocytosis (17) (Figure 1).

\subsection{Exosome and Liver Diseases}

In the complex environment of the liver, various types of cells, including hepatocytes, cholangiocytes, HSCs, LSECs, natural killer T cells (NKT), Kuffer cells, and adult liver stem cells can either release or receive exosomes in

Copyright (c) 2017, Hepatitis Monthly. This is an open-access article distributed under the terms of the Creative Commons Attribution-NonCommercial 4.0 International License (http://creativecommons.org/licenses/by-nc/4.0/) which permits copy and redistribute the material just in noncommercial usages, provided the original work is properly cited. 


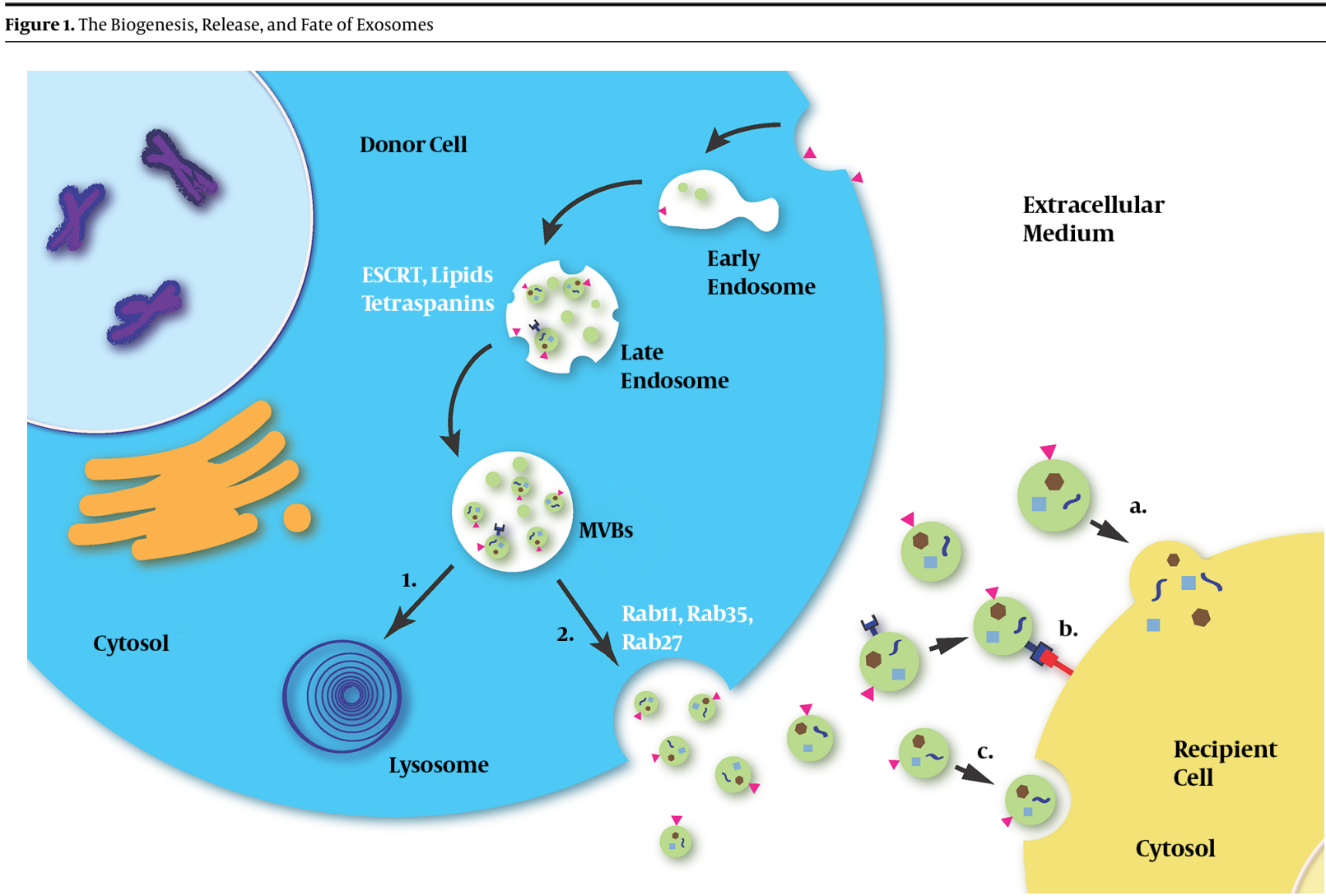

Exosomes are released by the donor cells and then captured by the recipient cells. Initially, early endosomes are produced by cellular endocytosis and develop into late endosome; subsequently, small vesicles, which are called intraluminal vesicles (ILVs), are generated by inward budding from the limiting membrane of endocytic vesicles (both early and late exosomes); and finally, ILVs develop into multivesicular bodies (MVBs). This process involves ESCRT, lipids, tetraspanins, etc. MVBs can either fuse with lysosomes for degradation or fuse with plasma membranes and release ILVs into the extracellular milieu. Rab proteins (Rab11, RAB35 and Rab27) are involved in the fusion of MVBs with the plasma membrane and the release of exosomes. After exosomes arrive at recipient cells, they exert a message-delivering function in one of three ways: a) through fusion with the plasma membrane; b) through ligand-receptor interaction; and c) via an endocytosis-dependent pathway.

cell-cell communication (18). Due to their newly discovered functions in inter-cellular information exchange, exosomes play an important role in liver physiology and pathology (Figure 2).

\section{Evidence Acquisition}

In this study we collected and summarized the most important and new data available on the role of exosome in liver diseases by the PubMed search. The study data were collected through searching the related keywords: exosome, liver, liver disease, liver cancer, hepatocellular carcinoma, HCC, cholangiocarcinoma, CCA, hepatitis, hepatitis $\mathrm{C}$ virus, $\mathrm{HCV}$, hepatitis $\mathrm{B}$ virus, $\mathrm{HBV}$, alcoholic hepatitis, $\mathrm{AH}$, non-alcoholic steatohepatitis, NASH, non-alcohol fatty liver disease, and NAFLD.

\section{Results}

\subsection{Exosomes in Liver Physiology and Pathology Processes}

Most of the cells in the liver have the capability to release and receive exosomes. For example, mouse hepatocytes can secrete exosomes that contain the cytochrome P450, UDP-glucuronosyltransferases (UDPGT), and glutathione S-transferases; in humans, these exosomes may exist in the plasma and play a role in extra-hepatic detoxification (19). Exosomes derived from rat bile can suppress the proliferation of cholangiocytes by inhibiting the ERK signaling pathway and increasing the expression of miR15a (20). Prostaglandin E2-carrying exosomes from intestinal epithelial cells can inhibit the activation of liver NKT cells via $\alpha$-GalCer and weaken the inflammatory responseinduced liver injury (21).

Exosomes and their content also contribute to liver regeneration. Hiroyuki et al. discovered that exosomes derived from hepatocytes can promote the proliferation 


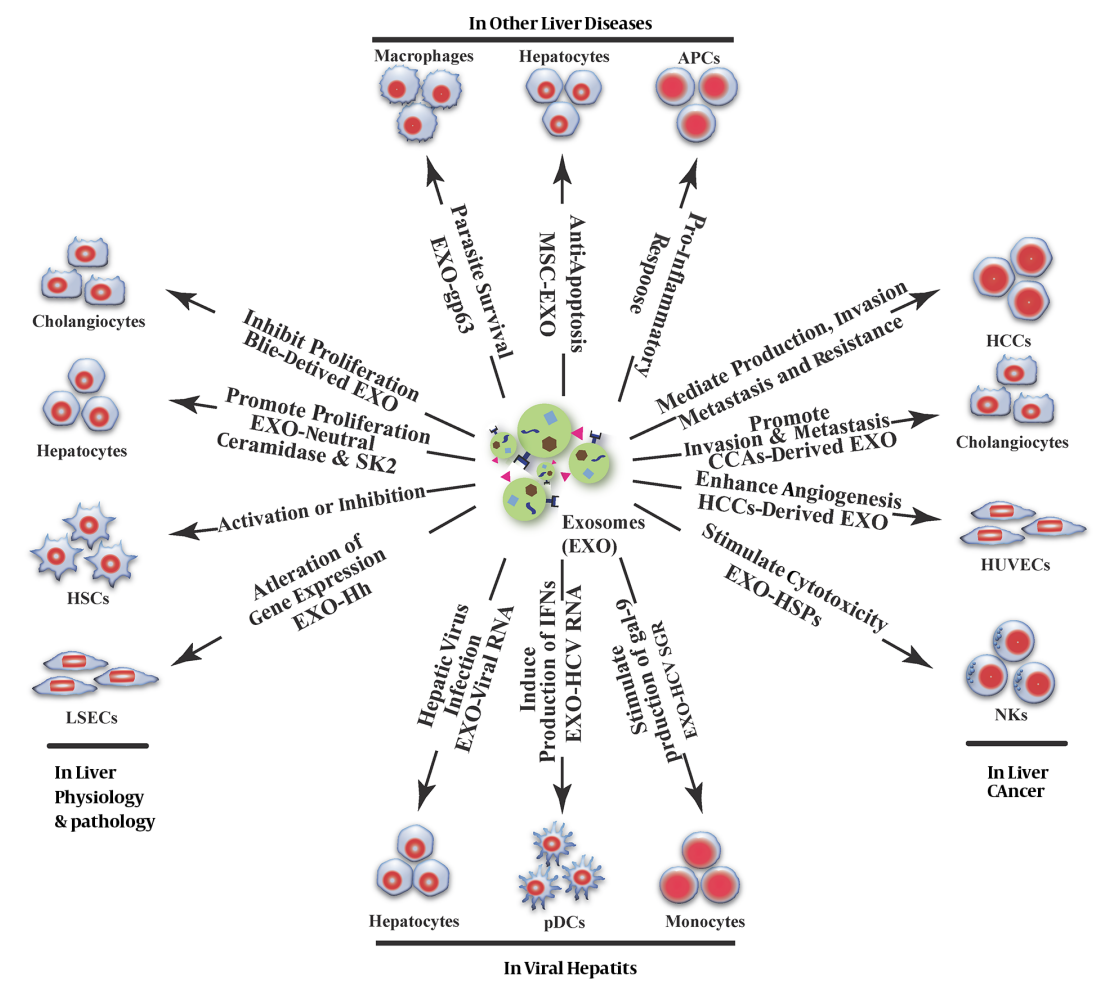

During physiological and pathological liver processes, exosomes from different sources have different functions, such as inhibiting cholangiocyte proliferation, promoting the proliferation of liver cells, activating HSCs or inhibiting the activated HSCs, and participating in liver fibrosis. In addition, exosomes can alter gene expression in LSECs and participate in liver cirrhosis. In viral hepatitis, exosomes are involved in the transmission of hepatitis viruses between cells; in addition, viral RNA-carrying exosomes participate in the regulation of immune responses. In liver cancer, tumor-derived exosomes are associated with tumorigenesis, tumor progression, invasion, angiogenesis, and drug resistance through various pathways, such as stimulating the anti-tumor response of NKcells. In other liver diseases (e.g., alcoholic liver disease or alcoholic hepatitis, drug-induced liver injury, non-alcoholic fatty liver disease or non-alcoholic steatohepatitis and primary biliary cirrhosis, exosomes can mediate pro-inflammatory responses and cause liver injury; however, mesenchymal stem cell (MSC)-derived exosomes can protect liver cells from hepatotoxicity. In parasitic liver disease, exosomes participate in adjusting the living conditions of parasites to facilitate their survival.

and regeneration of liver tissue both in vitro and in mice models of liver ischemia/reperfusion injury or partial hepatectomy (22). The researchers believed that the underlying mechanism involves sphingosine 1-phosphate (S1P). Hepatocyte-derived exosomes carrying neutral ceramidase and sphingosine kinase 2 (SK2) are captured by adjacent hepatocytes and raise the intracellular level of sphingosine-1-phosphate, thus promoting hepatocyte proliferation (22).

Hepatic fibrosis is a vital stage in the process of liver pathology and is commonly present in various chronic liver diseases. Patients with advanced hepatic fibrosis often require liver transplantation. Pathogenesis of the liver involves various types of liver cells; of these, HSCs are the key link in the occurrence and development of hepatic fibrosis. LSEC-derived exosomes containing sphingosine kinase 1 (SK1) can adhere to and then enter HSCs after bind- ing to HSCs through the fibronectin/ $\alpha 5 \beta 1$-integrin complex; afterwards, the exosomes result in an enhanced migration ability of target HSCs via the SK1/S1P pathway, thus participating in liver fibrosis (17). Moreover, simultaneous, with the above-described process, the activated HSCs can secrete exosomes that carry a high level of a connective tissue growth factor (CCN2), which in turn augments the fibrosis signal and activates more HSCs to accelerate the liver fibrosis process through interaction with other components (23). In contrast, quiescent HSCs can release exosomes that deliver Twist1 (a 21-kDa bHLH transcription factor) (24) and miR-214 (25) to activated HSCs. Twist1 can bind to DNM3os (noncoding dynamin 3 opposite strand transcript) E-box and promote miR-214 expression. miR-214 not only directly binds to CCN2 mRNA 3' -UTR and inhibits its activity, it also suppresses the expression of CCN2 downstream molecules such as alpha smooth muscle actin and 
collagen. As a result, the function of the activated HSCs in liver fibrosis is inhibited $(24,25)$. This might be a new mechanism underlying signal controlling in liver fibrosis (Figure 3).

Moreover, hepatic fibrosis is subject to the effects of exosomes derived from extra-hepatic cells. Exosomes secreted from human umbilical mesenchymal stem cells (HUCMSCs) can inhibit epithelial-tomesenchymal transition and decrease type I and II collagen deposition, thus alleviating CCl4-induced mouse liver fibrosis (26). miR-125bcarrying exosomes released from chorionic plate-derived mesenchymal stem cells (CP-MSCs) can reach the activated HSCs and inhibit the expression of Smo and other target genes to block the Hedgehog signaling pathway, which is also a contributor to liver fibrosis, thus ultimately alleviating liver fibrosis (27) (Figure 3). Myofibroblastic hepatic stellate cells and cholangiocytes treated with platelet-derived growth factor can secret exosomes that carry bioactive Hedgehog ligands. The same type of exosomes is also found in the plasma and bile of mice with ligated bile ducts. The ingested exosomes in LSEC can alter the expression of related genes in cells and participate in the pathological process of liver cirrhosis caused by biliary obstruction (28). Although the role of exosomes in physiological and pathological changes in the liver is still not entirely clear, these reports suggest that various exosomes have an unneglectable role. Furthermore, utilization of a particular substance or exosomes from specific origins to alter hepatic pathology might be a feasible treatment method to slow down the progression of liver fibrosis and cirrhosis.

\subsection{Exosomes in Viral Hepatitis}

\subsubsection{HCV}

The hepatitis $\mathrm{C}$ virus (HCV) is a single-strand RNA virus that causes acute and chronic hepatitis. Patients with longterm HCV infections develop a variety of complications, including liver fibrosis, cirrhosis, and even HCC. Previous research has confirmed the presence of viral RNA in the plasma exosomes of patients with HCV infection (29), although, no sufficient evidence has proven the role of exosomes in mediating infection. However, a series of recent studies revealed that exosomes derived from the HCVinfected Huh7.5 cells and plasma of HCV-infected patients contained full-length viral RNA, viral protein or virus particles, which allowed the exosomes to infect adjacent cells effectively (30-34). Cells containing HCV subgenomic replicon (SGR), which lack viral structural protein and cannot produce virus particles are able to release exosomes carrying subgenomic HCV RNA; subsequently, the exosomes are ingested by target cells leading to viral replication in the cells $(31,32)$. This indicates a viral particle-independent pathway for HCV transmission.

In addition, the relationship between HCV RNAcarrying exosomes and the human immune system has been gradually clarified. For instance, HCV-infected hepatocytes can release exosomes packaged with full-length viral RNA $(35,36)$ or HCV-SGR $(37)$, which are then transported to and activate plasmacytoid dendritic cells (pDCs), ultimately triggering interferon production. HCV-RNA exosomes secreted from hepatocytes with HCV or HCV-SGR infection can stimulate the production of galectin- 9 in monocytes (38). The increase in galectin-9 may be related to the suppression of the autoimmune response, which in turn causes the HCV infection to be persistent (38). In summary, it is very likely that HCV RNA-containing exosomes contribute to the HCV propagation as well as infection in the host and are related to the innate immune response induced by HCV. Moreover, it is speculated that these exosomes are involved in the pathological processes of $\mathrm{HCV}$-induced chronic hepatitis and the resulting cirrhosis as well as HCC.

In the extracellular environment, exosomes act as not only a vehicle but also a space that protects the HCV viral particles or viral RNA. Exosomes can protect HCV RNA from being broken down by extracellular RNase. Moreover, they provide an additional pathway for viruses or viral RNA to enter cells. This pathway is independent from CD81, scavenger receptor type1, apolipoprotein E receptor, and HCV E1/E2 proteins (viral structural proteins), resulting in the poor efficacy of immunotherapy for HCV treatment (34). Researchers have found that miR-122, HSP90, and Ago2 complex in exosomes can enhance HCV replication, suggesting that the utilization of miR-122 and HSP9O inhibitors can inhibit exosome-mediated HCV infection (34). In addition, the same research group discovered that proton pump inhibitors or bafilomycin A1 (vacuolartype H+-ATPase inhibitor) can change intracellular $\mathrm{pH}$ and damage the endocytic function of the cells, thus suppressing exosome-mediated HCV infection (34). Shrivastava et al. confirmed that the inhibition of autophagy of HCVinfected hepatocytes can result in an intracellular accumulation of HCV proteins and nucleic acids resulting from a reduced exosome-mediated HCV release (39). Exosomes derived from type I/III type interferon (IFN)-treated SEC cell line TMNK-1 cells can inhibit HCV replication in infected Huh7.5 cells, which may be attributed to an upregulation of antiviral gene expression (40). In the study of Li et al. IFN- $\alpha$-induced liver non-parenchymal cells (LNPCs) released exosomes containing antiviral molecules, which led to the suppression of HCV replication (41). Pan et al. used exosomes as a CD81 siRNA carrier to suppress CD81 expression, providing a potential exosome-related RNA interfer- 


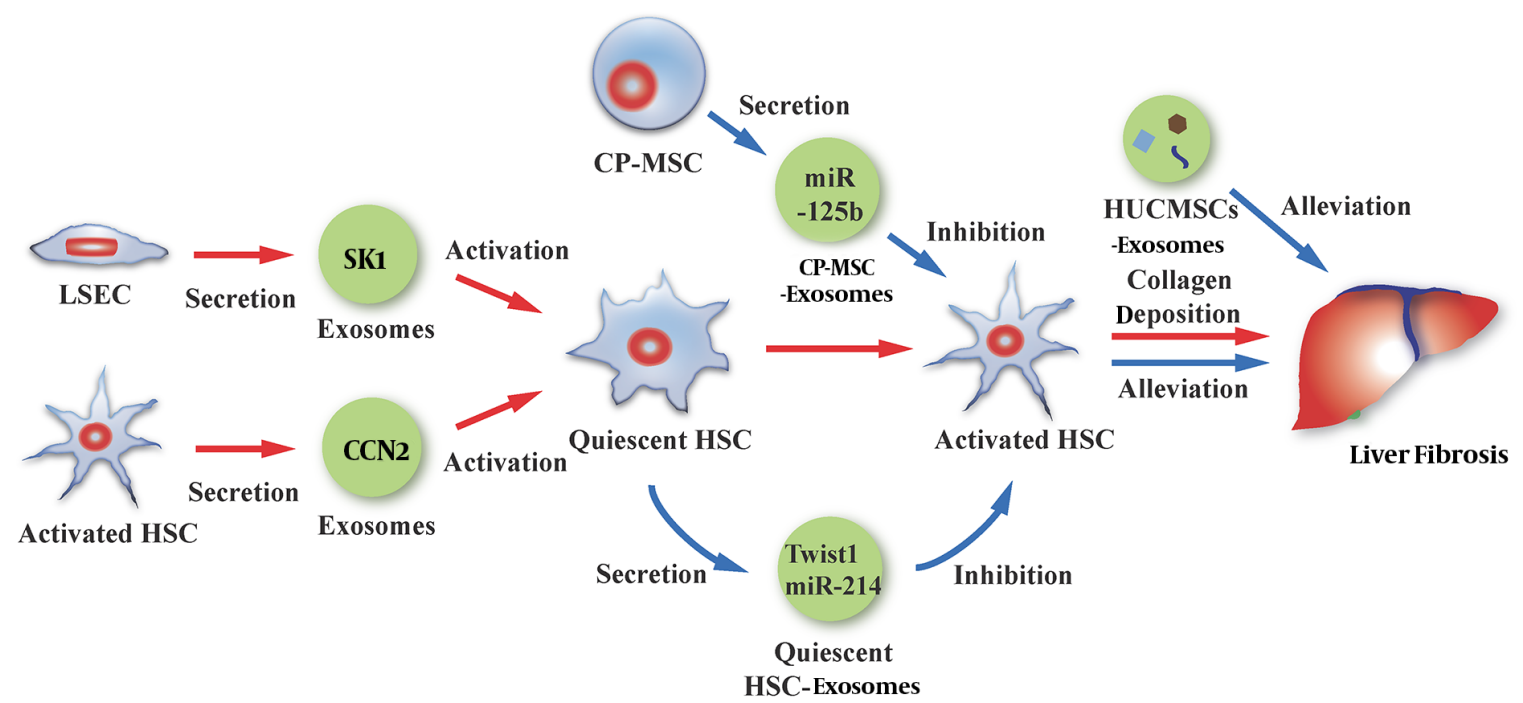

HSC plays an important role in the process of hepatic fibrosis. On the one hand, LSEC exosomes or activated HSC exosomes can participate in liver fibrosis by activating quiescent HSCs. On the other hand, quiescent HSC exosomes or CP-MSC exosomes can alleviate liver fibrosis by inhibiting the activity of activated HSCs. In addition, HUCMSCs exosomes contribute to the suppression of liver fibrosis.

ence therapy for HCV (42). All of these studies shed some light on new therapeutic strategies for blocking HCV transmission.

\subsubsection{Other Types of Viral Hepatitis}

HBV infection can significantly change the protein content of the exosomes from Huh-7 cells, e.g., the elevated level of valosin-containing protein (VCP, transitional endoplasmic reticulum ATPase). Due to the fact that the VCP increase in the liver is associated with the postoperative recurrence of HCC, it is speculated that alterations of the VCP level might be related to HBV-induced HCC (43). Li et al. revealed that IFN- $\alpha$-induced LNPCs (e.g., macrophages and LSECs) can secrete antiviral molecules such as APOBEC3G (a cellular cytidine deaminase), certain types of mRNA (IFITM1 and GTPBP2), and miRNA (miR-638, miR-4284, and miR-1260) to hepatocytes as well as suppress the replication of HBV, using exosomes as carriers. Moreover, after ingesting the exosomes, the recipient HBV-infected hepatocytes return to an antiviral state (41).

Some evidences also suggest that exosomes might contribute to immune neutralization escape of other types of hepatitis viruses. HAV, a non-enveloped virus, has been found in extracellular exosome-like vesicles and can evade the body's immune system and enter the cells (44). In in vitro experiments, HGV (human pegivirus, HPgV) RNAcontaining exosome-like vesicles are positive for exosomal marker protein CD63 and can deliver viral RNA to peripheral blood mononuclear cells (PBMCs), where HGV RNA can replicate (45).

\subsection{Exosomes in Non-Viral Hepatitis}

In various liver injury models induced by alcohol, drug, inflammation, or diet, the changes in the protein contents as well as mRNA levels/types in the exosomes have been proven to be satisfactory indicators for evaluating inflammation and promising biomarkers for detecting liver injury (46-53).

An earlier study reported that the serum exosomes contained higher levels of miR-122 and miR-155 in mice with alcohol- or Toll-like receptor (TLR) 9 ligand (CpG motif, cytidine-phosphate-guanosine)/TLR4 ligand (LPS, lipopolysaccharide)-induced liver injury (46). MomenHeravi et al. found that the exosome level and the miR-192, miR-30a, and miR-122 levels in exosomes were significantly higher in the circulation system of alcohol-fed mice and alcoholic hepatitis (AH) patients, with the miR-192 level showing the greatest increase (47). Similarly, a significant elevation of the miR-122 level was reported in another 
study of alcohol-treated mice, healthy alcoholic individuals, alcohol-treated Huh7.5 cells, and primary liver cells (48). In the AH patients, the remarkably increased number of exosomes in their circulation system is comprised mostly of exosomes derived from alcohol-exposed monocytes, which contain a significantly elevated level of miR27a. The exosomal miR-27a can stimulate naïve monocytes to differentiate into M2 macrophages, increase the secretion of IL-10, and transforming growth factor $\beta$, thus enhancing the phagocytic capacity (49). Recently, Vikas et al. found that alcohol-exposed hepatocytes can release CD40 ligand (CD40L, a member of the tumor necrosis factor protein family) containing extracellular vesicles or exosomes via a caspase dependent pathway, however, not dependent of apoptosis, which activate macrophage and promote it to produce pro-inflammatory factors, responsible for inflammation in alcoholic liver disease (54). In an in vitro experiment using monocytic THP1 cells, the miR-122 in the exosomes derived from alcohol-treated hepatocytes acted on the related targets on the THP1 cells and induced a proinflammatory response (48).

In mouse models of liver injury induced by acetaminophen (APAP) and D-galactosamine, the liverspecific mRNA level in the serum exosomes increased (55). Bala et al. found that miR-122 increased and miR-155 slightly decreased in the exosomes in an APAP-induced liver injury model (46). Likewise, hepatocyte-derived exosomes showed significantly increased levels of liverspecific RNAs albumin and miR-122 to sub-toxic APAP exposure in an early step of liver injury, researchers who maintained these exosomes may be associated to early immune responses in the formation of drug-induced liver injury (56). In in vitro experiments, significant protein changes were observed in exosomes secreted by hepatotoxicant-treated hepatocytes and similar changes were also detected in the serum exosomes in a rat model of liver injury induced by galactosamine, e.g., the levels of carboxylesterase (CES) protein family members and CES3 significantly increased (51). The injection of the exosomes derived from the peripheral blood of mice that were fed a high-fat diet resulted in an accumulation of immature myeloid cells (CD11b + Ly6C + Ly6C-cells) in the liver of the recipient mice, which might be responsible for obesityrelated liver disease (57). In a diet-induced non-alcoholic steatohepatitis mouse model (in which the mice were fed a methionine-choline-deficient diet), the injured liver cells released miR-122 into the circulatory system via exosomes and thus decreased the miR-122 level in the liver (53).

Despite the involvement of exosomes in liver injury, exosomes with specific origins might contribute to the alleviation of liver cell injury, thus protecting the liver. For example, in in vitro studies, exosomes have been used as vehicles for RNA interference to inhibit the miR-122-mediated proinflammatory response of macrophages (48). MSC-derived exosomes can improve the abilities of liver cells to resist drug-induced injury and apoptosis and promote cell proliferation and tissue regeneration (58). Such exosomes can stimulate the expression of related genes and proliferative proteins (such as proliferating cell nuclear antigen and cyclin D1) in hepatocytes, thus promoting cell cycle progression and increasing cell proliferation. For APAP- or $\mathrm{H}_{2} \mathrm{O}_{2}$ induced liver injury, MSC-derived exosomes can upregulate the expression of Bcl-xL protein to suppress toxicantinduced apoptosis (58).

\subsection{Exosomes in Liver Cancer}

3.4.1. The Role of Exosomes in Tumorigenesis and the Development of Liver Cancer

The role of exosomes in many tumors has been explored as the unique structural and functional features of exosomes have drawn increased attention from researchers. A recent study done by Hoshino et al. verified the "seed-and-soil" theory of cancer occurrence and progression proposed by the British physician Stephen Pagent. Hoshino et al. believed that exosomes might act as "seeds" for tumor metastasis. Moreover, they stated that the ability to target tumor metastasis may be associated with a specific integrin family in exosomes (59). In the study done by Kogure et al. the transfer of some miRNAs into HepG2 through an uptake of Hep3B-derived exosomes resulted in the suppression of transforming growth factor $\beta$ activated kinase-1(TAK1), and this loss of TAK1 activity was believed to be associated with hepatocarcinogenesis (60). In another recent report, exosomes derived from HCC cell line SMMC-7721 cells can in turn promote the growth and proliferation, metastasis, and invasion of the cells themselves (61). In addition, the exosomes released from highly migratable HCC cell lines (HKCI-C3, HKCI-8 and MHCC97L) carry an abundant amount of protumorigenic RNA and proteins, including MET protooncogene, S100 family members (S100A4, S100A10, and S100A11), and caveolins (CAV-1 and (AV-2), which increase the production of active matrix metalloproteinases (MMP)-2 as well as MMP-9 by activating the PI13K/AKT and MAPK signaling pathways, thus significantly enhancing the ability of non-motile hepatocyte cell line to migrate and invade (62). Similar studies were also conducted for other liver tumors. For example, for cholangiocarcinoma (CCA), an in vitro study revealed that the exosomes from CCA cell line KKU-M213 cells carry oncogenic proteins that increase the migration and invasion of the normal biliary epithelial $\mathrm{H} 69$ cells (63).

HepG2-derived exosomes contain vasorin, which can enhance the migration of human umbilical vein endothelial cells (HUVECs) (64). CD90+ liver cancer cells (known 
as cancer stem cell-like cells, CSC cells) can release such vasorin -carrying exosomes to increase the production of vascular endothelial growth factor (VEGF), its receptor VEGFR1, and intercellular cell adhesion molecule-I in HUVECs, thus promoting the adhesion of endothelial cells and the formation of tube-like structures (65). Moreover, long-noncoding RNA(IncRNA) in the exosomes plays a role in the development of liver tumors. CD90+ Huh7-derived exosomes carrying lncRNA H19 might contribute to HCC intrahepatic metastasis and angiogenesis (65). The intracellular transportation of linc-RoR via exosomes can improve the survival of HCC cells under hypoxic conditions (66). In summary, exosomes play a crucial role in liver tumor tumorigenesis, progression, migration, as well as angiogenesis, and contribute to the construction of tumor microenvironment.

\subsubsection{Exosomes as Biological Markers}

It may be possible to use particular contents of exosomes as biological markers to predict the diagnosis and prognosis of HCC. The level and type of miRNA in the exosomes found in the serum of HCC patients differ significantly the miRNA in the serum exosomes of normal healthy individuals (67-70). For instance, HCC patients with larger tumors and those with recurrent HCC after liver transplantation had lower levels of miR-718 in serumderived exosomes. The down-regulation of miR-718 can lead to HOXB8 overexpression, which might be associated with the poor prognosis of these patients (69). Moreover, using a diethylnitrosamine-treated mouse model, Liu et al. found that considering a combination of specific miRNAs changes in the serum and exosomes improved the effectiveness and accuracy of HCC diagnosis in mice, especially at the early stage of cirrhosis (70).

\subsubsection{Roles of Exosomes in Hepatic Tumor Growth Suppression and Treatment Response Improvement}

Exosomes not only play a specific role in inhibiting hepatic tumor cell proliferation, migration, and invasion; they also affect the treatment response of tumors. HCC cells treated with anticancer drugs can release exosomes containing HSPs, such as HSP60, HSP70, and HSP90, which can induce the cytotoxic responses of NK cells and the subsequent anti-tumor immune response (71). In the study done by Fonsato et al., human liver stem cell-derived exosomes carrying anti-tumor miRNAs (miR-451 and miR-31) suppressed the growth of HCC in with severe combined immune deficiency (SCID) mice that had been inoculated with primary HCC (72). Human macrophages can also transmit miRNA to HCC cells to inhibit cell proliferation via exosomes (73). Recent research revealed that exosomes containing Vps4A (a tumor suppressor) can reduce the proliferation, metastasis, and invasion of HCC cells (61). In addition, under stress conditions, tumor cells can release exosomes to adapt themselves to the surrounding environment. For example, chemotherapy with sorafenib, camptothecin, and doxorubicin might significantly elevate the level of linc-VLDLR, a long non-coding RNA, in HCCsecreted exosomes, which in turn improves the proliferative activity and drug resistance of the nearby recipient cells (74). However, in a study conducted by Sudarshana et al. after Huh7 cell-derived miR-122-carring exosomes were captured by HepG2 cells, they suppressed cell growth and proliferation, accelerated cells' senescence, and increased the sensitivity of cells to doxorubicin (75). Similarly, in another study that involved both in vivo and in vitro experiments, the use of exosomes produced by adult mesenchymal stem cells as vehicles to transport miR-122 to HCC cells remarkably enhanced the sensitivity of tumor cells to sorafenib and 5-FU (76).

In conclusion, the discovery of exosomes provides a new perspective on the tumorigenesis and progression of liver cancer. With this increase in knowledge, exosomebased technologies can offer both a promising tool for the diagnosis, a prognostic prediction of cancer, and a new strategy for liver cancer treatment.

\subsection{Exosomes in Other Liver Diseases}

A number of studies have also revealed the involvement of exosomes in liver injury caused by other pathogenic factors. In primary biliary cirrhosis patients, circulating exosomes can regulate the expression of co-stimulatory molecules in antigen presenting cells (APCs), thus contributing to the development of primary biliary cirrhosis (77). Ghosh et al. found that Leishmania donovani-infected host cells (macrophages) could secret exosomes carrying gp63, a $\mathrm{Zn}^{2+}$ dependent metalloprotease. After exosomes were endocytosed by Huh 7 cells, the virulence factor gp63 was released to cleave Dicer1, resulting in a decrease of mature miR-122 in the cells, which led to a decline in the serum cholesterol level and ultimately benefited parasite survival and infection (78).

\section{Conclusions}

The discovery of exosomes opens up new horizons for information transmission between cells and provides new knowledge regarding the development of disease. More importantly, it offers a promising new direction for disease diagnosis and treatment. In recent years, an increasing number of studies have explored the role of exosomes in liver disease. These studies revealed the involvement of exosomes in various aspects of liver physiology and pathology and in the progress of liver diseases. Moreover, the 
potential application of exosomes in liver disease diagnosis as well as treatment and prognosis prediction was preliminarily explored. The findings confirmed the undeniable role of exosomes as a new pathway for information exchange in liver diseases. However, despite the considerable progress that related studies have made, research on the relationship between exosomes and liver disease is still in the initial stage. A number of questions remain to be addressed, such as the mechanisms underlying exosome biogenesis, the sorting mechanisms that take place under the specific conditions (e.g., the packing of virus RNAs into exosomes in HBV-infected hepatocytes), exosome secretion, exosome targeting in the extracellular environment, and the role of exosomes in liver tumor occurrence, invasion, angiogenesis, as well as microenvironment construction. In addition, in-depth research should be conducted to expand the clinical application of exosomes and their contents as valuable biomarkers for disease diagnosis and monitoring or as excellent carriers to deliver drugs for targeted therapy in liver diseases.

\section{Footnotes}

Authors' Contribution: Gang Chen, Pengyi Guo, and Yi Wang designed the review article. Haitao Yu and Xiaozai Xie drafted the manuscript.

Financial Disclosure: The authors have no potential conflicts of interest.

Funding/Support: Authors received no financial support or grant for this review.

\section{References}

1. Ohno S, Ishikawa A, Kuroda M. Roles of exosomes and microvesicles in disease pathogenesis. Adv Drug Deliv Rev. 2013;65(3):398-401. doi: 10.1016/j.addr.2012.07.019. [PubMed: 22981801].

2. Goetzl EJ, Boxer A, Schwartz JB, Abner EL, Petersen RC, Miller BL, et al. Altered lysosomal proteins in neural-derived plasma exosomes in preclinical Alzheimer disease. Neurology. 2015;85(1):40-7. doi: 10.1212/WNL.0000000000001702. [PubMed: 26062630]

3. O'Brien K, Rani S, Corcoran C, Wallace R, Hughes L, Friel AM, et al. Exosomes from triple-negative breast cancer cells can transfer phenotypic traits representing their cells of origin to secondary cells. Eur J Cancer. 2013;49(8):1845-59. doi: 10.1016/j.ejca.2013.01.017. [PubMed: 23453937].

4. Lowry MC, Gallagher WM, O'Driscoll L. The Role of Exosomes in Breast Cancer. Clin Chem. 2015;61(12):1457-65. doi: 10.1373/clinchem.2015.240028. [PubMed: 26467503].

5. Brinton LT, Sloane HS, Kester M, Kelly KA. Formation and role of exosomes in cancer. Cell Mol Life Sci. 2015;72(4):659-71. doi: 10.1007/s00018-014-1764-3. [PubMed: 25336151].

6. Zhang X, Yuan X, Shi H, Wu L, Qian H, Xu W. Exosomes in cancer: small particle, big player.J Hematol Oncol. 2015;8:83. doi: 10.1186/s13045-0150181-x. [PubMed: 26156517].

7. Yu S, Cao H, Shen B, Feng J. Tumor-derived exosomes in cancer progression and treatment failure. Oncotarget. 2015;6(35):37151-68. doi: 10.18632/oncotarget.6022. [PubMed: 26452221].
8. Colombo M, Raposo G, Thery C. Biogenesis, secretion, and intercellular interactions of exosomes and other extracellular vesicles. Annu Rev Cell Dev Biol. 2014;30:255-89. doi: 10.1146/annurev-cellbio-101512122326. [PubMed: 25288114]

9. Johnstone RM, Adam M, Hammond JR, Orr L, Turbide C. Vesicle formation during reticulocyte maturation. Association of plasma membrane activities with released vesicles (exosomes). J Biol Chem. 1987;262(19):9412-20. [PubMed: 3597417].

10. Liu Y, Gu Y, Cao X. The exosomes in tumor immunity. Oncoimmunology. 2015;4(9):e1027472. doi: 10.1080/2162402X.2015.1027472. [PubMed: 26405598].

11. Lo Cicero A, Stahl PD, Raposo G. Extracellular vesicles shuffling intercellular messages: for good or for bad. Curr Opin Cell Biol. 2015;35:6977. doi: 10.1016/j.ceb.2015.04.013. [PubMed: 26001269].

12. Cocucci E, Meldolesi J. Ectosomes and exosomes: shedding the confusion between extracellular vesicles. Trends Cell Biol. 2015;25(6):364-72. doi: 10.1016/j.tcb.2015.01.004. [PubMed: 25683921].

13. Keerthikumar S, Chisanga D, Ariyaratne D, Al Saffar H, Anand S, Zhao K, et al. ExoCarta: A Web-Based Compendium of Exosomal Cargo. J Mol Biol. 2016;428(4):688-92. doi: 10.1016/j.jmb.2015.09.019. [PubMed 26434508].

14. Minciacchi VR, Freeman MR, Di Vizio D. Extracellular vesicles in cancer: exosomes, microvesicles and the emerging role of large oncosomes. Semin Cell Dev Biol. 2015;40:41-51. doi: 10.1016/j.semcdb.2015.02.010. [PubMed: 25721812].

15. Segura E, Guerin C, Hogg N,Amigorena S, Thery C.CD8+ dendritic cells use LFA- 1 to capture MHC-peptide complexes from exosomes in vivo.J Immunol. 2007;179(3):1489-96. [PubMed:17641014].

16. Montecalvo A, Larregina AT, Shufesky WJ, Stolz DB, Sullivan ML, Karls son JM, et al. Mechanism of transfer of functional microRNAs between mouse dendritic cells via exosomes. Blood. 2012;119(3):756-66. doi: 10.1182/blood-2011-02-338004. [PubMed: 22031862].

17. Wang R, Ding Q, Yaqoob U, de Assuncao TM, Verma VK, Hirsova P, et al. Exosome Adherence and Internalization by Hepatic Stellate Cells Triggers Sphingosine 1-Phosphate-dependent Migration. J Biol Chem. 2015;290(52):30684-96. doi: 10.1074/jbc.M115.671735. [PubMed: 26534962].

18. Masyuk AI, Masyuk TV, Larusso NF. Exosomes in the pathogenesis, diagnostics and therapeutics of liver diseases. J hepatol. 2013;59(3):621-

19. Conde-Vancells J, Rodriguez-Suarez E, Embade N, Gil D, Matthiesen $\mathrm{R}$, Valle $\mathrm{M}$, et al. Characterization and comprehensive proteome profiling of exosomes secreted by hepatocytes. J Proteome Res. 2008;7(12):5157-66. [PubMed:19367702].

20. Masyuk AI, Huang BQ, Ward CI, Gradilone SA, Banales JM, Masyuk $\mathrm{TV}$, et al. Biliary exosomes influence cholangiocyte regulatory mechanisms and proliferation through interaction with primary cilia Am J Physiol Gastrointest Liver Physiol. 2010;299(4):G990-9. doi 10.1152/ajpgi.00093.2010. [PubMed: 20634433].

21. Deng ZB, Zhuang X, Ju S, Xiang X, Mu J, Liu Y, et al. Exosome-like nanoparticles from intestinal mucosal cells carry prostaglandin E2 and suppress activation of liver NKT cells. J Immunol. 2013;190(7):3579-89. doi: 10.4049/jimmunol.1203170. [PubMed: 23467936].

22. Nojima H, Freeman CM, Schuster RM, Japtok L, Kleuser B, Edwards MJ, et al. Hepatocyte exosomes mediate liver repair and regeneration via sphingosine-1-phosphate. J Hepatol. 2016;64(1):60-8. doi: 10.1016/j.jhep.2015.07.030. [PubMed: 26254847].

23. Charrier A, Chen R, Chen L, Kemper S, Hattori T, Takigawa M, et al Exosomes mediate intercellular transfer of pro-fibrogenic connective tissue growth factor (CCN2) between hepatic stellate cells, the principal fibrotic cells in the liver. Surgery. 2014;156(3):548-55. doi: 10.1016/j.surg.2014.04.014. [PubMed: 24882759]. 
24. Chen L, Chen R, Kemper S, Charrier A, Brigstock DR. Suppression of fibrogenic signaling in hepatic stellate cells by Twist1-dependent microRNA-214 expression: Role of exosomes in horizontal transfer of Twist1. Am J Physiol Gastrointest Liver Physiol. 2015;309(6):G491-9. doi: 10.1152/ajpgi.00140.2015. [PubMed: 26229009].

25. Chen L, Charrier A, Zhou Y, Chen R, Yu B, Agarwal K, et al. Epigenetic regulation of connective tissue growth factor by MicroRNA-214 delivery in exosomes from mouse or human hepatic stellate cells. Hepatol ogy. 2014;59(3):1118-29. doi: 10.1002/hep.26768. [PubMed: 24122827]

26. Li T, Yan Y, Wang B, Qian H, Zhang X, Shen L, et al. Exosomes derived from human umbilical cord mesenchymal stem cells alleviate liver fibrosis. Stem Cells Dev. 2013;22(6):845-54. doi: 10.1089/scd.2012.0395. [PubMed: 23002959].

27. Hyun J, Wang S, Kim J, Kim GJ, Jung Y. MicroRNA125b-mediated Hedgehog signaling influences liver regeneration by chorionic plate-derived mesenchymal stem cells. Sci Rep. 2015;5:14135. doi: 10.1038/srep14135. [PubMed: 26370741].

28. Witek RP, Yang L, Liu R, Jung Y, Omenetti A, Syn WK, et al. Liver cell-derived microparticles activate hedgehog signaling and alter gene expression in hepatic endothelial cells. Gastroenterology. 2009;136(1):320-330 e2. doi: 10.1053/j.gastro.2008.09.066. [PubMed: 19013163].

29. Masciopinto F, Giovani C, Campagnoli S, Galli-Stampino L, Colombatto P, Brunetto M, et al. Association of hepatitis $C$ virus envelope proteins with exosomes. Eur J Immunol. 2004;34(10):2834-42. doi: 10.1002/eji.200424887. [PubMed: 15368299].

30. Cosset FL, Dreux M. HCV transmission by hepatic exosomes establishes a productive infection. J Hepatol. 2014;60(3):674-5. doi: 10.1016/j.jhep.2013.10.015. [PubMed: 24512825].

31. Ramakrishnaiah V, Thumann C, Fofana I, Habersetzer F, Pan Q, de Ruiter PE, et al. Exosome-mediated transmission of hepatitis $\mathrm{C}$ virus between human hepatoma Huh7.5 cells. Proc Natl Acad Sci U S A. 2013;110(32):13109-13. doi: 10.1073/pnas.1221899110. [PubMed: 23878230].

32. Longatti A, Boyd B, Chisari FV. Virion-independent transfer of replication-competent hepatitis $C$ virus RNA between permissive cells. J Virol. 2015;89(5):2956-61. doi: 10.1128/JVI.02721-14. [PubMed: 25505060].

33. Liu Z, Zhang X, Yu Q, He JJ. Exosome-associated hepatitis C virus in cell cultures and patient plasma. Biochem Biophys Res Commun. 2014;455(3-4):218-22. doi: 10.1016/j.bbrc.2014.10.146. [PubMed: 25449270].

34. Bukong TN, Momen-Heravi F, Kodys K, Bala S, Szabo G. Exosomes from hepatitis $C$ infected patients transmit $\mathrm{HCV}$ infection and contain replication competent viral RNA in complex with Ago2miR122-HSP90. PLoS Pathog. 2014;10(10):e1004424. doi: 10.1371/journal.ppat.1004424. [PubMed: 25275643].

35. Dreux M, Garaigorta U, Boyd B, Decembre E, Chung J, Whitten-Bauer $\mathrm{C}$, et al. Short-range exosomal transfer of viral RNA from infected cells to plasmacytoid dendritic cells triggers innate immunity. Cell Host Microbe. 2012;12(4):558-70. doi: 10.1016/j.chom.2012.08.010. [PubMed: 23084922].

36. Nakai M, Oshiumi H, Funami K, Okamoto M, Matsumoto M, Seya T, et al. Interferon (IFN) and Cellular Immune Response Evoked in RNAPattern Sensing During Infection with Hepatitis C Virus (HCV). Sensors (Basel). 2015;15(10):27160-73. doi: 10.3390/s151027160. [PubMed: 26512676].

37. Bobrie A, Colombo M, Raposo G, Thery C. Exosome secretion: molecular mechanisms and roles in immune responses. Traffic. 2011;12(12):1659-68. doi: 10.1111/j.1600-0854.2011.01225.x. [PubMed: 21645191].

38. Harwood NM, Golden-Mason L, Cheng L, Rosen HR, Mengshol JA. HCVinfected cells and differentiation increase monocyte immunoregulatory galectin-9 production. J Leukoc Biol. 2016;99(3):495-503. doi: 10.1189/jlb.5A1214-582R. [PubMed: 26475932].

39. Shrivastava S, Devhare P, Sujijantarat N, Steele R, Kwon YC, Ray R, et al. Knockdown of Autophagy Inhibits Infectious Hepatitis C Virus Release by the Exosomal Pathway. J Virol. 2015;90(3):1387-96. doi: 10.1128/JVI.02383-15. [PubMed: 26581990].

40. Giugliano S, Kriss M, Golden-Mason L, Dobrinskikh E, Stone AE, Soto-Gutierrez A, et al. Hepatitis $C$ virus infection induces autocrine interferon signaling by human liver endothelial cells and release of exosomes, which inhibits viral replication. Gastroenterology. 2015;148(2):392-402 e13. doi: 10.1053/j.gastro.2014.10.040. [PubMed: 25447848].

41. Li J, Liu K, Liu Y, Xu Y, Zhang F, Yang H, et al. Exosomes mediate the cellto-cell transmission of IFN-alpha-induced antiviral activity. Nat Immunol. 2013;14(8):793-803. doi: 10.1038/ni.2647. [PubMed: 23832071].

42. Pan Q, Ramakrishnaiah V, Henry S, Fouraschen S, de Ruiter PE, Kwekkeboom J, et al. Hepatic cell-to-cell transmission of small silencing RNA can extend the therapeutic reach of RNA interference (RNAi). Gut. 2012;61(9):1330-9. doi: 10.1136/gutjnl-2011-300449. [PubMed: 22198713].

43. Zhao X, Wu Y, Duan J, Ma Y, Shen Z, Wei L, et al. Quantitative proteomic analysis of exosome protein content changes induced by hepatitis $\mathrm{B}$ virus in Huh-7 cells using SILAC labeling and LC-MS/MS.JProteome Res. 2014;13(12):5391-402. doi: 10.1021/pr5008703. [PubMed: 25265333].

44. Feng Z, Hensley L, McKnight KL, Hu F, Madden V, Ping L, et al. A pathogenic picornavirus acquires an envelope by hijacking cellular membranes. Nature. 2013;496(7445):367-71. doi:10.1038/nature12029. [PubMed: 23542590].

45. Chivero ET, Bhattarai N, Rydze RT, Winters MA, Holodniy M, Stapleton JT. Human pegivirus RNA is found in multiple blood mononuclear cells in vivo and serum-derived viral RNA-containing particles are infectious in vitro. J Gen Virol. 2014;95(Pt 6):1307-19. doi: 10.1099/vir.0.063016-0. [PubMed: 24668525].

46. Bala S, Petrasek J, Mundkur S, Catalano D, Levin I, Ward J, et al. Circulating microRNAs in exosomes indicate hepatocyte injury and inflammation in alcoholic, drug-induced, and inflammatory liver diseases. Hepatology. 2012;56(5):1946-57. doi: 10.1002/hep.25873. [PubMed: 22684891].

47. Momen-Heravi F, Saha B, Kodys K, Catalano D, Satishchandran A Szabo G. Increased number of circulating exosomes and their microRNA cargos are potential novel biomarkers in alcoholic hepatitis. J Transl Med. 2015;13:261. doi: 10.1186/s12967-015-0623-9. [PubMed: 26264599].

48. Momen-Heravi F, Bala S, Kodys K, Szabo G. Exosomes derived from alcohol-treated hepatocytes horizontally transfer liver specific miRNA-122 and sensitize monocytes to LPS. Sci Rep. 2015;5:9991. doi: 10.1038/srep09991. [PubMed: 25973575].

49. Saha B, Momen-Heravi F, Kodys K, Szabo G. MicroRNA Cargo of Extracellular Vesicles from Alcohol-exposed Monocytes Signals Naive Monocytes to Differentiate into M2 Macrophages. I Biol Chem. 2016;291(1):149-59. doi: 10.1074/jbc.M115.694133. [PubMed: 26527689].

50. Yang X, Weng Z, Mendrick DL, Shi Q. Circulating extracellular vesicles as a potential source of new biomarkers of drug-induced liver injury. Toxicol Lett. 2014;225(3):401-6. doi: 10.1016/j.toxlet.2014.01.013. [PubMed: 24462978].

51. Rodriguez-Suarez E, Gonzalez E, Hughes C, Conde-Vancells J, Rudella A, Royo F, et al. Quantitative proteomic analysis of hepatocytesecreted extracellular vesicles reveals candidate markers for liver toxicity. J Proteomics. 2014;103:227-40. doi: 10.1016/j.jprot.2014.04.008. [PubMed: 24747303].

52. Povero D, Eguchi A, Li H, Johnson CD, Papouchado BG, Wree A, et al. Circulating extracellular vesicles with specific proteome and liver microRNAs are potential biomarkers for liver injury in experimental fatty liver disease. PLoS One. 2014;9(12):e113651. doi: 10.1371/journal.pone.0113651. [PubMed: 25470250].

53. Csak T, Bala S, Lippai D, Satishchandran A, Catalano D, Kodys K, et al. microRNA-122 regulates hypoxia-inducible factor-1 and vimentin in hepatocytes and correlates with fibrosis in diet-induced steato- 
hepatitis. Liver Int. 2015;35(2):532-41. doi: 10.111/liv.12633. [PubMed: 25040043].

54. Verma VK, Li H, Wang R, Hirsova P, Mushref M, Liu Y, et al. Alcohol stimulates macrophage activation through caspase-dependent hepatocyte derived release of $\mathrm{CD} 40 \mathrm{~L}$ containing extracellular vesicles. $J$ Hepatol. 2016;64(3):651-60. doi: 10.1016/j.jhep.2015.11.020. [PubMed: 26632633].

55. Wetmore BA, Brees DJ, Singh R, Watkins PB, Andersen ME, Loy J, et al. Quantitative analyses and transcriptomic profiling of circulating messenger RNAs as biomarkers of rat liver injury. Hepatology. 2010;51(6):2127-39. doi: 10.1002/hep.23574. [PubMed: 20235334].

56. Holman NS, Mosedale M, Wolf KK, LeCluyse EL, Watkins PB. Subtoxic Alterations in Hepatocyte-Derived Exosomes: An Early Step in Drug-Induced Liver Injury? Toxicol Sci. 2016;151(2):365-75. doi: 10.1093/toxsci/kfw047. [PubMed: 26962055].

57. Deng ZB, Liu Y, Liu C, Xiang X, Wang J, Cheng Z, et al. Immature myeloid cells induced by a high-fat diet contribute to liver inflammation. Hepatology. 2009;50(5):1412-20. doi: 10.1002/hep.23148. [PubMed: 19708080].

58. Tan CY, Lai RC, Wong W, Dan YY, Lim SK, Ho HK. Mesenchymal stem cell-derived exosomes promote hepatic regeneration in druginduced liver injury models. Stem Cell Res Ther. 2014;5(3):76. doi: 10.1186/scrt465. [PubMed: 24915963].

59. Hoshino A, Costa-Silva B, Shen TL, Rodrigues G, Hashimoto A, Tesic Mark M, et al. Tumour exosome integrins determine organotropic metastasis. Nature. 2015;527(7578):329-35. doi: 10.1038/nature15756. [PubMed: 26524530].

60. Kogure T, Lin WL, Yan IK, Braconi C, Patel T. Intercellular nanovesiclemediated microRNA transfer: a mechanism of environmental modulation of hepatocellular cancer cell growth. Hepatology. 2011;54(4):1237-48. doi: 10.1002/hep.24504. [PubMed: 21721029].

61. Wei JX, Lv LH, Wan YL, Cao Y, Li GL, Lin HM, et al.Vps4A functions as a tumor suppressor by regulating the secretion and uptake of exosomal microRNAs in human hepatoma cells. Hepatology. 2015;61(4):1284-94. doi: 10.1002/hep.27660. [PubMed: 25503676].

62. He M, Qin H, Poon TC, Sze SC, Ding X, Co NN, et al. Hepatocellular carcinoma-derived exosomes promote motility of immortalized hepatocyte through transfer of oncogenic proteins and RNAs. Carcinogenesis. 2015;36(9):1008-18. doi: 10.1093/carcin/bgv081. [PubMed: 26054723].

63. Dutta S, Reamtong O, Panvongsa W, Kitdumrongthum S, Janpipatkul $\mathrm{K}$, Sangvanich $\mathrm{P}$, et al. Proteomics profiling of cholangiocarcinoma exosomes: A potential role of oncogenic protein transferring in cancer progression. Biochim Biophys Acta. 2015;1852(9):1989-99. doi: 10.1016/j.bbadis.2015.06.024. [PubMed: 26148937].

64. Huang A, Dong J, Li S, Wang C, Ding H, Li H, et al. Exosomal transfer of vasorin expressed in hepatocellular carcinoma cells promotes migration of human umbilical vein endothelial cells. Int J Biol Sci. 2015;11(8):961-9. doi: 10.7150/ijbs.11943. [PubMed: 26157350].

65. Conigliaro A, Costa V, Lo Dico A, Saieva L, Buccheri S, Dieli F, et al. CD90+ liver cancer cells modulate endothelial cell phenotype through the release of exosomes containing H19 IncRNA. Mol Cancer. 2015;14:155. doi: 10.1186/s12943-015-0426-x. [PubMed: 26272696].

66. Takahashi K, Yan IK, Haga H, Patel T. Modulation of hypoxia-signaling pathways by extracellular linc-RoR. J Cell Sci. 2014;127(Pt 7):1585-94. doi: 10.1242/jcs.141069. [PubMed: 24463816].

67. Wang H, Hou L, Li A, Duan Y, Gao H, Song X. Expression of serum exosomal microRNA-21 in human hepatocellular carcinoma. Biomed Res Int. 2014;2014:864894. doi: 10.1155/2014/864894. [PubMed: 24963487].

68. Sohn W, Kim J, Kang SH, Yang SR, Cho JY, Cho HC, et al. Serum exosomal microRNAs as novel biomarkers for hepatocellular carcinoma. Exp Mol Med. 2015;47:e184. doi: 10.1038/emm.2015.68. [PubMed: 26380927].

69. Sugimachi K, Matsumura T, Hirata H, Uchi R, Ueda M, Ueo H, et al Identification of a bona fide microRNA biomarker in serum exosomes that predicts hepatocellular carcinoma recurrence after liver transplantation. Br J Cancer. 2015;112(3):532-8. doi: 10.1038/bjc.2014.621. [PubMed: 25584485].

70. Liu WH, Ren LN, Wang X, Wang T, Zhang N, Gao Y, et al. Combination of exosomes and circulating microRNAs may serve as a promising tumor marker complementary to alpha-fetoprotein for earlystage hepatocellular carcinoma diagnosis in rats. J Cancer Res Clin Oncol. 2015;141(10):1767-78. doi: 10.1007/s00432-015-1943-0. [PubMed: 25724413].

71. Lv LH, Wan YL, Lin Y, Zhang W, Yang M, Li GL, et al. Anticancer drugs cause release of exosomes with heat shock proteins from human hepatocellular carcinoma cells that elicit effective natural killer cell antitumor responses in vitro. J Biol Chem. 2012;287(19):15874-85. doi 10.1074/jbc.M112.340588. [PubMed: 22396543].

72. Fonsato V, Collino F, Herrera MB, Cavallari C, Deregibus MC, Cisterna $\mathrm{B}$, et al. Human liver stem cell-derived microvesicles inhibit hepatoma growth in SCID mice by delivering antitumor microRNAs. Stem Cells. 2012;30(9):1985-98. doi:10.1002/stem.1161. [PubMed: 22736596].

73. Aucher A, Rudnicka D, Davis DM. MicroRNAs transfer from human macrophages to hepato-carcinoma cells and inhibit proliferation. IImmunol. 2013;191(12):6250-60. doi: 10.4049/jimmunol.1301728. [PubMed: 24227773].

74. Takahashi K, Yan IK, Wood J, Haga H, Patel T. Involvement of extracellular vesicle long noncoding RNA (linc-VLDLR) in tumor cell responses to chemotherapy. Mol Cancer Res. 2014;12(10):1377-87. doi: 10.1158/15417786.MCR-13-0636. [PubMed: 24874432]

75. Basu S, Bhattacharyya SN. Insulin-like growth factor-1 prevents miR122 production in neighbouring cells to curtail its intercellular transfer to ensure proliferation of human hepatoma cells. Nucleic Acids Res. 2014;42(11):7170-85. doi: 10.1093/nar/gku346. [PubMed: 24813441].

76. Lou G, Song X, Yang F, Wu S, Wang J, Chen Z, et al. Exosomes derived from miR-122-modified adipose tissue-derived MSCs increase chemosensitivity of hepatocellular carcinoma. $J$ Hematol Oncol. 2015;8:122. doi: 10.1186/s13045-015-0220-7. [PubMed: 26514126].

77. Tomiyama T, Yang GX, Zhao M, Zhang W, Tanaka H, Wang J, et al. The modulation of co-stimulatory molecules by circulating exosomes in primary biliary cirrhosis. Cell Mol Immunol. 2017;14(3):276-84. doi: 10.1038/cmi.2015.86. [PubMed: 26388238].

78. Ghosh J, Bose M, Roy S, Bhattacharyya SN. Leishmania donovani targets Dicer1 to downregulate miR-122, lower serum cholesterol, and facilitate murine liver infection. Cell Host Microbe. 2013;13(3):277-88. doi: 10.1016/j.chom.2013.02.005. [PubMed: 23498953]. 